\title{
Ausência de assimetria vertical na orientação encoberta da atenção visual
}

\author{
Vertical asymmetry absence on covert orientation of visual attention \\ Ausencia de asimetría vertical en la orientación encubierta de la atención visual
}

Recebido: 21/07/2021 | Revisado: 26/07/2021 | Aceito: 28/07/2021 | Publicado: 04/08/2021

\author{
Daniela Pires Abrão Galindo \\ ORCID: https://orcid.org/0000-0003-4435-6431 \\ Universidade do Oeste Paulista, Brasil \\ E-mail: daneabrao@gmail.com \\ Bianca Lika Date \\ ORCID: https://orcid.org/0000-0002-5044-3980 \\ Universidade do Oeste Paulista, Brasil \\ E-mail: biancalika_date@ hotmail.com \\ Felipe Viegas Rodrigues \\ ORCID: https://orcid.org/0000-0002-1073-867X \\ Universidade do Oeste Paulista, Brasil \\ E-mail: rodrigues.fv@gmail.com
}

\begin{abstract}
Resumo
A atenção é um conjunto de processos que leva à seleção ou priorização da informação, tem como objetivo filtrar as informações evitando a sobrecarga perceptual no processamento de estímulos. Diversas funções cerebrais são lateralizadas como a fala e o processamento espacial. A orientação da atenção tem maior processamento no hemisfério direito, com favorecimento à direita do espaço visual. O presente estudo objetivou investigar assimetrias verticais da atenção. Para tal, foi utilizada uma adaptação do Paradigma da Atenção Encoberta de Posner (1980) com pistas endógenas. Participaram do experimento trinta e seis sujeitos, homens e mulheres saudáveis. Os resultados mostraram ausência de assimetria vertical na orientação encoberta da atenção visual, tanto para os tempos de reação, quanto para a porcentagem de erros. Há uma possível assimetria no SOA mais longo, com discreto favorecimento do campo visual superior. Futuros trabalhos devem investigar se a simetria na orientação da atenção visuoespacial é suportada por ativação neural também equivalente, diferentemente do que acontece para a orientação da atenção no plano horizontal. Palavras-chave: Atenção; Cognição; Lateralidade funcional; Psicofísica.
\end{abstract}

\begin{abstract}
Attention is as a set of processes leading to the selection or prioritization of information, filtering the latter and avoiding perceptual overload in the processing of stimuli. Several brain functions are lateralized such as speech and spatial processing. The orientation of attention is more processed in the right hemisphere, favoring the right visual space. The present study aimed to investigate vertical asymmetries of attention. For this purpose, an adaptation of the Posner's Attention Paradigm (1980) with central clues was used. Thirty-six subjects participated in the experiment, healthy men and women. The results showed no vertical asymmetry in the covert orientation of visual attention, both for reaction times and for the percentage of errors. There is a possible asymmetry in the longer SOA, with a slight favoring of the superior visual field. Future work should investigate whether symmetry in the orientation of visuospatial attention is supported by equivalent neural activation, differently from what happens for the orientation of attention in the horizontal plane.
\end{abstract}

Keywords: Attention; Cognition; Functional laterality; Psychophysics.

\section{Resumen}

La atención es un conjunto de procesos que conducen a la selección o priorización de información, con el objetivo de filtrar la información, evitando la sobrecarga perceptiva en el procesamiento de los estímulos. Varias funciones cerebrales están lateralizadas, como el habla y el procesamiento espacial. La orientación de la atención se procesa más en el hemisferio derecho, favoreciendo el espacio visual derecho. El presente estudio tuvo como objetivo investigar las asimetrías verticales de atención. Para ello se utilizó una adaptación del Paradigma de Atención Encubierta de Posner (1980) con pistas endógenas. Treinta y seis sujetos participaron en el experimento, hombres y mujeres sanos. Los resultados no mostraron asimetría vertical en la orientación encubierta de la atención visual, tanto para los tiempos de reacción como para el porcentaje de errores. Existe una posible asimetría en la SOA más larga, con un ligero favorecimiento del campo visual superior. El trabajo futuro debería investigar si la simetría en la orientación de la atención visuoespacial está respaldada por una activación neural equivalente, a diferencia de lo que sucede con la orientación de la atención en el plano horizontal.

Palabras clave: Atención; Cognición; Lateralidad funcional; Psicofísica. 


\section{Introdução}

A atenção é investigada desde o século XIX, conceituada por William James (1890, p.375) em "Os Princípios de Psicologia", como "a ação de tomar posse realizada pelo espírito, de forma clara e vívida, de um entre outros vários objetos ou séries de pensamentos simultaneamente possíveis. Focalização, concentração da consciência são sua essência. Implica o afastamento de algumas coisas para ocupar-se efetivamente de outras". Assim sendo, a atenção é um mecanismo de seleção de informações relevantes ao comportamento, associado à cognição (Angelopoulou \& Drigas, 2021).

O sistema nervoso possui especializações hemisféricas, também presentes em outras espécies além dos seres humanos, como camundongos e primatas (Corballis, 2007). O hemisfério esquerdo, por exemplo, é dominante em pessoas destras, e responsável pelas funções de fala e escrita, pela realização de cálculos matemáticos e leitura, sendo nomeado como o centro primário da linguagem. Por sua vez, o hemisfério direito é específico para o reconhecimento facial, solução de problemas espaciais, percepção social, raciocínio simbólico e desempenhos artísticos; é predominante na função de perceber e analisar modelos visuais (Badzakova-Trajkov et al., 2010). Há sugestões antigas de que estas diferenças podem ter surgido como uma solução para o crescimento do encéfalo e a necessidade de manter comunicação rápida entre diferentes regiões corticais, que pode ser lenta entre os hemisférios cerebrais (Ringo et al., 1994). Em suma, o hemisfério esquerdo está relacionado à memória verbal, e o direito às localizações espaciais, fisionomias e melodias (Gazzaniga, 1997; Wagner, 2003; Guardiola, 1998).

A orientação da atenção também parece assimétrica, tanto no tempo (Nobre, 2001), quanto no espaço (Castro-Barros et al., 2008). Brain (1941) foi o primeiro a observar a relação entre perda parcial da visão esquerda e uma incapacidade de orientar a atenção para este lado do espaço, denominada heminegligência contralateral, em três pacientes com lesão do lobo parietal e occipital direito. Segundo Mesulam (1999), essa condição faz com que o paciente perca a capacidade de notar estímulos relevantes à esquerda, e assim, todos os estímulos se tornam insignificantes e serão ignorados. Isso pode ocorrer sem que haja perda de visão, e essa incapacidade pode estar associada tanto à visão, quanto à audição ou ao tato (Gainotti, 2010).

A assimetria das redes neurais da atenção foi demonstrada em estudos com ressonância magnética funcional (fMRI, do inglês Functional Magnetic Resonance Imaging), que observou maior ativação do hemisfério direito em tarefas de atenção (Corbetta et al.,1993, Nobre et al.,1997, Kim et al.,1999). Corbetta et al. (1993), submeteram participantes a um teste de atenção no qual alvos eram dispostos sequencialmente ao lado direito e esquerdo do campo visual. O estudo demonstrou que alvos apresentados no hemicampo visual direito, levam a maior ativação de córtices parietal e frontal superior esquerdo e córtex temporal superior direito, porém em relação ao hemicampo esquerdo, houve ativação bilateral de córtices parietal superior (de maior intensidade direita), parietal inferior direito e frontal superior direito. Esses resultados sugerem que na orientação da atenção, há uma rede frontoparietal dorsal bilateral assimétrica envolvendo também, circuitos ventrais de hemisfério direito, na junção têmporo parietal. Apesar dessa assimetria de ativação neural, os autores relataram ausência de diferenças entre os tempos de reação para alvos à direita ou à esquerda do hemicampo visual.

Jewell e McCourt (2000) mostraram evidências de que maior quantidade de recursos neurais no hemisfério direito favorece a orientação da atenção para este lado. Participantes saudáveis expostos ao teste de bissecção de linhas, i.e, instruídos a dividir ao meio linhas horizontais de diferentes tamanhos, o fazem ligeiramente à esquerda, por supervalorização do espaço à direita, o que ficou conhecido como pseudonegligência. De fato, Castro-Barros, Righi, Grechi e Ribeiro-do-Valle (2008) demonstraram favorecimento do espaço à direita nos tempos de reação no Paradigma da Atenção Encoberta de Posner (1980). As razões para o favorecimento à direita do espaço não são claras, mas poderiam estar relacionadas a treinos gerados pelo próprio ambiente, como a leitura comumente realizada da esquerda para a direita. Se este é o caso, é possível que outros favorecimentos possam existir, como para o lado inferior em relação ao superior, embora não se encontre na literatura trabalhos que tenham investigado especificamente essa possível assimetria. 
O Paradigma da Atenção Encoberta de Posner (1980) é um teste amplamente utilizado em estudos da orientação da atenção. Este analisa o efeito do direcionamento encoberto da atenção visual, em relação ao tempo de reação (RT) à estímulos exibidos no campo de visão, apresentados em um monitor de vídeo. Com o olhar fixo em um ponto da tela, o participante deve responder a informações projetadas à direita ou à esquerda, sendo que este receberá pistas sinalizadoras quanto a sua posição, que podem estar corretas, incorretas ou serem ausentes, podendo ainda ser exógenas (periférica, diretamente ao redor do alvo) ou endógenas (central, no campo periférico do alvo). Foi demonstrado que o RT é menor quando o indivíduo é indicado de maneira correta quanto à localização do alvo, em comparação à sinalização nula (ausente) ou errônea (Sumi, 2011; Rodrigues, 2011).

Os resultados deste paradigma sugerem que a sinalização correta facilita a resposta, devido à orientação da pista. Em relação à incorreta, se faz necessário a inibição da atenção ao campo visual errôneo e reorientação ao certo, havendo atraso na resposta. (Nahas \& Xavier, 2004) Além disso, por meio deste teste é possível comparar atenção automática e voluntária, sendo que a orientação automática da atenção ocorre em estímulos súbitos, que facilitam o processamento de informações e geram rápido efeito de atenção que não podem ser voluntariamente inibidos. Já a orientação voluntária da atenção é induzida pelo próprio indivíduo, sendo mais lenta que o anterior e pode ser inibida (Jonides, 1981).

Rafal, Posner, Friedman, Inhoff e Bernstein (1988) apresentaram quatro posições de alvos (acima, abaixo, à direita e à esquerda) e pistas endógenas e exógenas a oito pacientes com paralisia supranuclear progressiva (PSP) e oito pacientes com doença de Parkinson idiopática (DPI). Os resultados mostraram que, para ambos os grupos, os erros cometidos para os planos verticais e horizontais não foram diferentes e não houve efeitos significantes em relação à posição (vertical ou horizontal) do alvo. Porém, para ambos os grupos e ambas as pistas, o RT foi menor para pistas válidas, o efeito de validade da pista foi menor no plano vertical, e a latência da mudança de atenção foi maior no mesmo plano, sendo também maior para pistas exógenas. A diferença de orientação vertical comparada com a horizontal entre os dois grupos foi discretamente maior para pistas exógenas. Assim, mediante as patologias dos sujeitos, os autores também demonstraram a importância do mesencéfalo na regulação da atenção visual.

Dessa forma, o objetivo do presente trabalho é investigar a ocorrência de possíveis assimetrias verticais na orientação encoberta da atenção visual.

\section{Metodologia}

\subsection{Amostra}

O presente trabalho é analítico, experimental, longitudinal e quantitativo. Todos os procedimentos adotados com os participantes foram autorizados pelo CONEP via Plataforma Brasil (CAAE: 88358418.8.0000.5515). Participaram da pesquisa quarenta universitários, homens $(n=18)$ e mulheres $(n=22)$. A idade mínima dos participantes foi de 18 anos e a máxima de 35 anos. A abordagem dos participantes foi aleatória (dentre os limites de idade esperados), no próprio campus da universidade. Uma vez que aceitaram participar do experimento, foram convidados para a sala onde realizaram os experimentos. O questionário de Edinburgh (Oldfield, 1971) foi aplicado para avaliar a saúde física e mental dos sujeitos e conhecer características que validem os resultados dos testes que foram aplicados na sequência, como dominância manual, importante num teste que avalia assimetrias da atenção. Dos participantes selecionados, uma mulher foi excluída por erro na gravação dos dados do experimento e duas mulheres e um homem foram excluídos por intercorrências durante a realização do teste. Ao todo, contaram como resultados válidos dezessete homens $(n=17)$ e dezenove mulheres $(n=19)$. Nenhum participante tinha histórico de convulsão ou qualquer outro problema neurológico mais grave. 


\subsection{Teste de Atenção}

Foi utilizada uma variação do paradigma de atenção encoberta de Posner (1980), envolvendo pistas centrais preditivas ( $80 \%$ válidas e $20 \%$ inválidas) e alvos verticais, isto é, apresentados para cima e para baixo do ponto de fixação ao centro da tela, com indivíduos saudáveis (Figura 1). Para a geração dos estímulos e registro das respostas utilizou-se o software PsychoPy (Peirce et al., 2019) num computador rodando Windows®. Os participantes se sentaram à frente da tela, com a cabeça em um apoiador de fronte e mento, de tal forma que a distância entre os olhos e o ponto de fixação foi igual a $57 \mathrm{~cm}$, assim, $1 \mathrm{~cm}$ na tela corresponde a $1^{\circ}$ de ângulo visual. Os testes foram realizados numa sala de experimentos com atenuação acústica e iluminação reduzida.

Figura 1. O ponto de fixação é apresentado no centro da tela. Após um intervalo de $1000 \mathrm{~ms}$, a pista é apresentada por 62,50 ms. Após um SOA de 100, 300 ou 700 ms, o alvo com duração de 31,25 ms é apresentado. Os participantes dispõem de até 1000 ms para responder. O ponto de fixação é mantido na tela até o final desse tempo ou até que uma resposta seja apresentada. O intervalo entre as tentativas é de $350 \mathrm{~ms}$.

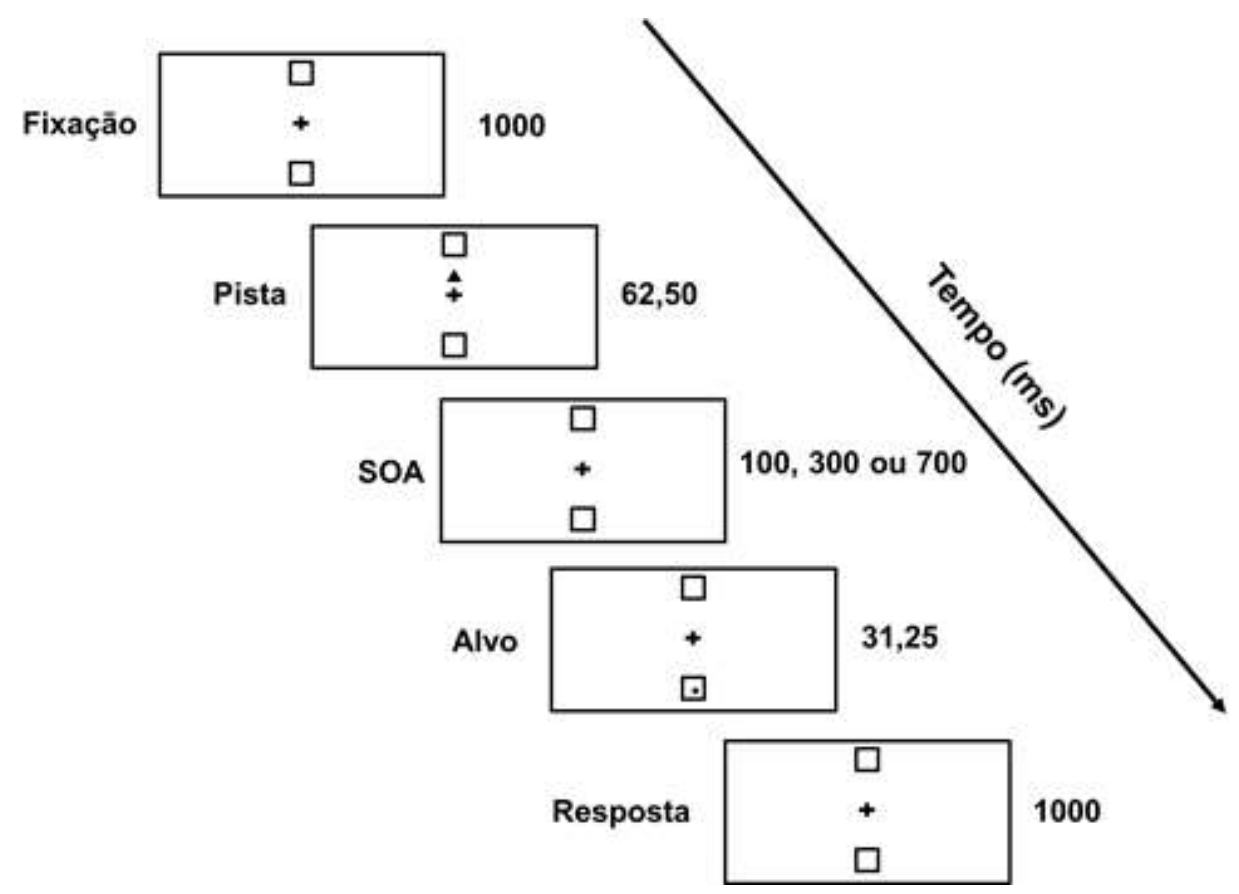

Fonte: Autores.

A cada tentativa: (1) o ponto de fixação foi apresentado no centro da tela; (2) após um intervalo de 1000 milissegundos, a pista foi apresentada por 62,50 ms (dois quadros com tela à 32Hz); (3) após um intervalo de tempo variável (SOA - do inglês "Stimulus Onset Asynchrony") que poderia ser 100, 300 ou 700 ms, (4) o alvo com duração de 31,25 ms foi apresentado (um quadro com tela à 32Hz). Os participantes dispuseram de até $1000 \mathrm{~ms}$ para responder. O ponto de fixação foi mantido na tela até o final desse tempo ou até que uma resposta fosse apresentada. O intervalo entre as tentativas foi de 350 ms. Os tempos de reação aos alvos apresentados na parte superior da tela foram acrescidos de um milissegundo, devido à diferença de tempo na apresentação dos alvos superiores e inferiores provocado pelo sincronismo vertical da tela (os pixels são alterados da parte superior para inferior). A diferença de tempo foi calculada a partir de uma filmagem em câmera superlenta da própria tela (à 960 quadros/s).

Os participantes foram instruídos a (1) manter o olhar no ponto de fixação, (2) de que a pista informava o provável local de aparecimento do alvo e a (3) responder ao alvo o mais rápido e precisamente possível, com a mão de preferência através da barra de espaços do teclado (e de que terão apenas $1000 \mathrm{~ms}$ para fazê-lo). Respostas emitidas até cem milissegundos 
após o aparecimento do alvo (incluindo os eventos anteriores da tentativa, i.e., apresentação do ponto de fixação, intervalo entre o ponto de fixação e a pista, apresentação da pista, SOA e apresentação do alvo) foram consideradas erros por antecipação. Após 1000 ms a ausência de resposta do sujeito foi registrada como um erro por omissão.

Os participantes eram informados pelo software sobre as tentativas em que estavam antecipando, numa tentativa de diminuir o número de antecipações. Cada sujeito respondeu a 300 tentativas aleatorizadas por SOA e por condição de validade, resultando em 50 tentativas para cada posição do alvo e para cada SOA. As 300 tentativas foram distribuídas em cinco blocos de 60 tentativas cada e, portanto, foram 240 tentativas válidas e 60 tentativas inválidas. Os intervalos de tempo entre os blocos foram livres, isto é, o participante poderia descansar pelo tempo que julgar conveniente; ele mesmo acionava uma tecla para dar prosseguimento às tentativas. $\mathrm{O}$ tempo total de teste foi de aproximadamente 15 minutos.

\subsection{Análises Estatísticas}

Os tempos de reação (RT) de cada participante considerando validade (válida e inválida), posição (extremidade superior ou inferior da tela) e SOA (100, 300 e $700 \mathrm{~ms})$ permitiram calcular a mediana dos RT em cada condição. Na análise geral, tendo sexo como fator entressujeitos, foi calculada a média dessas medianas, incluindo apenas as tentativas cujas respostas foram corretas. Foi escolhida a mediana para expressar o desempenho dos participantes, pois esta medida está menos sujeita à influência de escores extremos esporádicos que poderiam viesar a média, representando de forma mais fiel o desempenho dos sujeitos.

Para avaliar a acurácia da resposta foi calculada a porcentagem de respostas corretas. Análises de variância para medidas repetitivas (ANOVA) foram utilizadas para comparar os RT e a acurácia (porcentagem de respostas corretas em relação ao total de respostas possíveis), considerando sexo como fator entressujeitos e SOA, posição e validade como fatores intrassujeitos para os RTs e SOA e posição para a acurácia. Quando necessário, testes post-hoc Bonferroni foram realizados. Os gráficos foram todos confeccionados em python com o uso da biblioteca matplotlib (Hunter, 2007). Quando cabível, o indicador Omega squared $(\omega 2)$ foi utilizado para estimar o tamanho do efeito, sendo considerado insignificante quando $\omega 2<$ 0,01 , pequeno quando $0,01 \leq \omega 2<0,06$, médio para $0,06 \leq \omega 2<0,14$ e grande quando $\omega 2 \geq 0,14$ (Goss-Sampson, 2020), especialmente considerada a amostra pequena utilizada.

As análises estatísticas foram realizadas no software JASP (JASP Team, 2021), baseadas no modelo frequentista e com interpretação ponderada dos valores-p (Krueger \& Heck, 2019, Wasserstein \& Lazar, 2016), incluindo a recomendação de que as declarações de "significância estatística" sejam abandonadas (Wasserstein \& Lazar, 2019).

\section{Resultados}

\section{1 Tempos de reação}

A ANOVA para medidas repetidas mostrou efeito insignificante para a posição $(F 1,34=3,884 ; \mathrm{p}=0,057, \omega 2=0,004)$ e para a interação entre posição e SOA ( $F 2,68=3,221 ; \mathrm{p}=0,046, \omega 2=0,004)$ (Figura 2). A análise post-hoc mostrou diferença entre as posições somente no SOA $700(\mathrm{p}=0,033)$. Como esperado, a ANOVA mostrou efeito grande para SOA $(\mathrm{F} 2,68=81,966 ; \mathrm{p}<0,001, \omega 2=0,230)$ e efeito médio para validade ( $\mathrm{F} 1,34=54,402 ; \mathrm{p}<0,001, \omega 2=0,076)$. Interessantemente, há efeito pequeno para a interação entre validade e $\operatorname{SOA}(F 2,68=21,921 ; p<0,001, \omega 2=0,035)$. A análise da Figura 2 permite observar que o efeito de validade (EF) é reduzido no SOA 700. Não há diferença entre os sexos $(\mathrm{F} 1,34=0,994 ; \mathrm{p}=0,326$, $\omega 2=0,000$ ) ou suas interações. 
Figura 2. Tempos de reação no teste de Posner para alvos apresentados na posição superior e inferior da tela, por SOA, sexo e precedidos por pistas válidas ou inválidas. Barras representam Erro Padrão da Média (EPM).

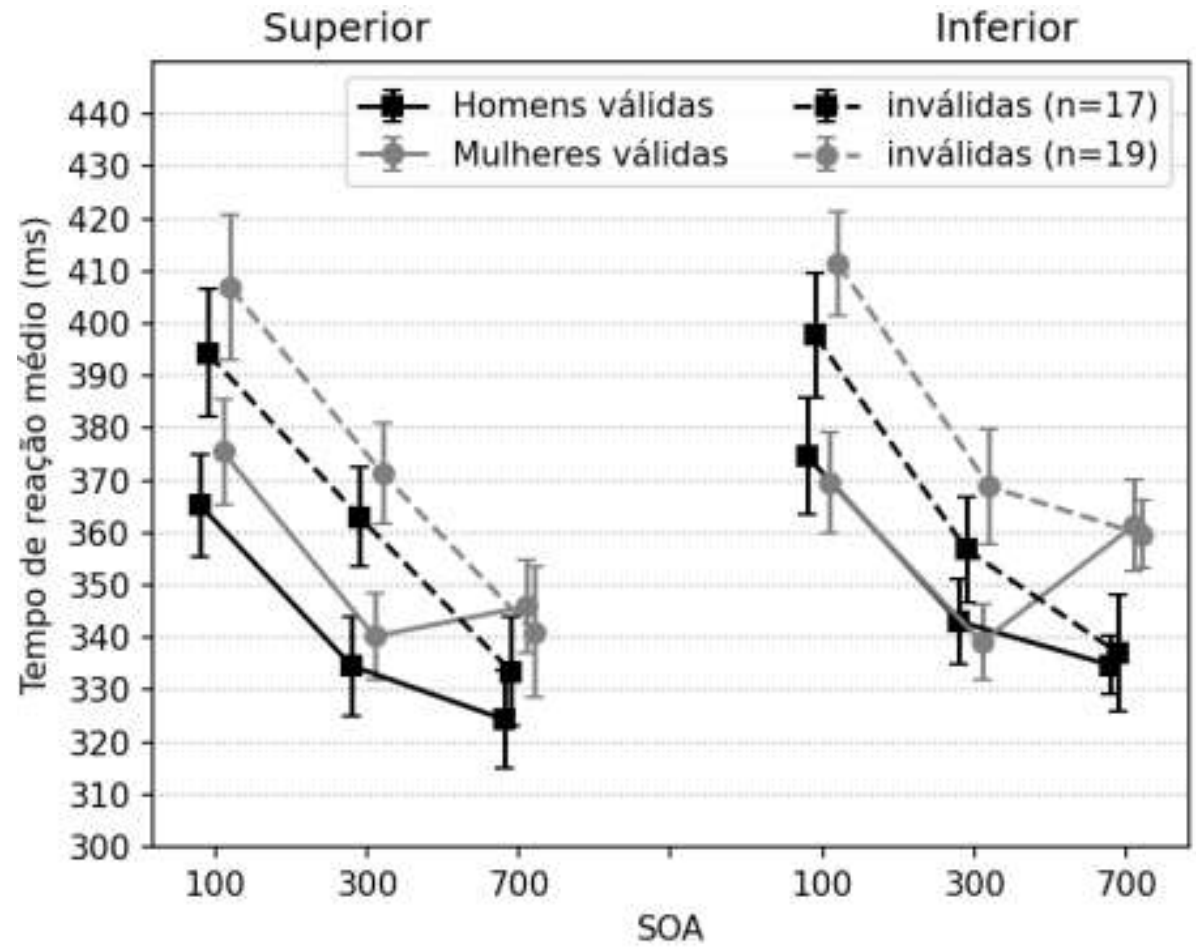

Fonte: Autores.

\subsection{Acurácia}

A ANOVA para medidas repetidas mostrou efeito insignificante para a posição $(F 1,34=0,012 ; p=0,913, \omega 2=0,000)$ e para a interação entre posição e $\mathrm{SOA}(\mathrm{F} 2,68=0,197 ; \mathrm{p}=0,822, \omega 2=0,000)$ (Figura 3). Novamente, há efeito grande para o $\mathrm{SOA}$ $(F 2,68=63,195 ; p<0,001, \omega 2=0,426)$ e não há diferença entre os sexos $(F 1,34=0,006 ; p=0,941, \omega 2=0,000)$ ou suas interações (Figura 3). Não há diferenças para os erros por omissão entre homens e mulheres $(F 1,34=0,001 ; p=0,977, \omega 2=0,000)$, que são apenas $0,61 \%$ do total de tentativas na média (Figura 3 ). 
Figura 3. Erros por omissão no teste de Posner para alvos apresentados na posição superior e inferior da tela, por SOA e sexo. Barras representam EPM.

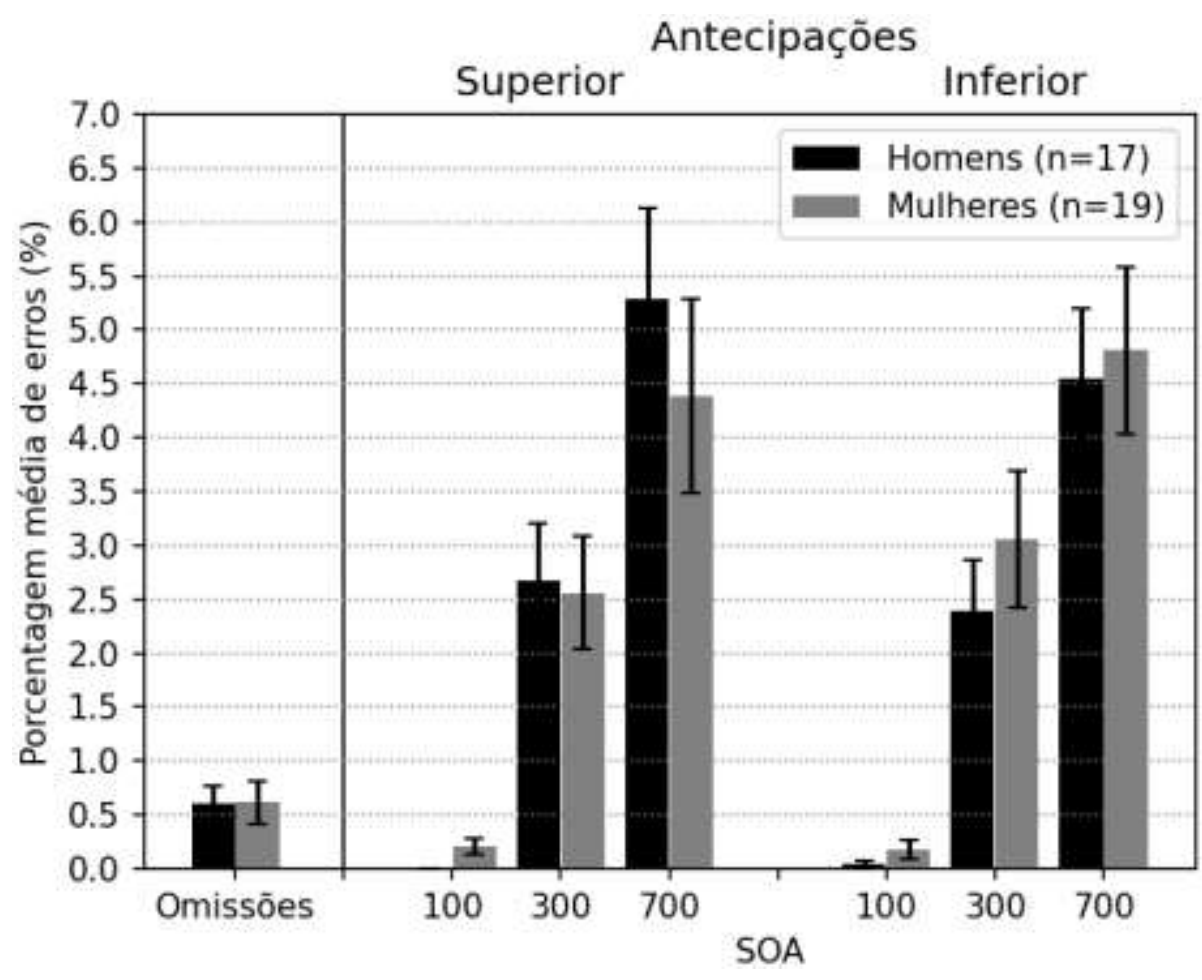

Fonte: Autores.

\section{Discussão}

O presente experimento evidenciou ausência de assimetria vertical na orientação encoberta da atenção visual em participantes jovens e saudáveis, num experimento realizado com pistas endógenas preditivas. Há uma possível assimetria no SOA mais longo, com discreto favorecimento do campo visual superior. Estes resultados devem ser confirmados por replicações deste experimento, preferencialmente com protocolos mais longos, com maior número de tentativas, já que no SOA mais longo acontece também o maior número de erros por antecipação. Estes dados complementam resultados anteriores da literatura que já haviam demonstrado assimetria horizontal na orientação encoberta da atenção endógena (Righi \& Ribeiro, 2011; Rodrigues, 2011).

Posner et al. (1982) e Rafal et al. (1988) investigaram assimetrias verticais da atenção anteriormente, mas não relataram evidência de assimetria da orientação encoberta da atenção, manifesta nos tempos de reação ou na acurácia aos alvos. Ambos os experimentos, no entanto, ocorreram com pacientes de paralisia supranuclear progressiva (PSP) (e também Parkinson, em Rafal et al., 1988). Posner et al. (1982) compararam as latências para efeito de validade em alvos verticais e horizontais e encontraram que a orientação da atenção ocorreu com maior latência no plano vertical. No plano horizontal o efeito da validade já se apresentou em SOAs rápidos, de apenas $50 \mathrm{~ms}$; no plano vertical nenhum efeito de orientação da atenção esteve presente até $1000 \mathrm{~ms}$ de SOA. Os autores também evidenciaram maior efeito de validade no plano horizontal. no presente trabalho o SOA mais longo utilizado foi de setecentos ms, o que dificulta comparações maiores com Posner $e t$ al. (1982). Interessantemente, mesmo os participantes incapazes de realizar sacadas no plano vertical ainda eram capazes de direcionar a atenção neste plano (encobertamente), com RTs maiores para pistas apresentadas na direção em que os pacientes possuíam o movimento ocular intacto, em relação a direção em que estes se encontravam afetados severamente, isto é, a sacada ocular atrasa a resposta do sujeito. 
Gómez, Flores e Digiacomo (2008) submeteram quatorze sujeitos saudáveis a alvos apenas no plano vertical, enquanto monitorados por meio de eletroencefalograma (EEG). Os resultados mostraram aumento da atividade cerebral nas regiões do córtex pré-frontal, córtex cingulado anterior em sua porção dorsal (DAAC - do inglês dorsal anterior cingulate cortex), pré-Cúneo e giro para-hipocampal. Curiosamente, a DAAC teve sua atividade ainda mais aumentada durante tentativas com pistas inválidas em relação às válidas, sugerindo que o processamento de pistas inválidas ativou áreas de monitoramento de conflitos, reforçando hipótese de que alvos precedentes de pistas inválidas produzem ativação cerebral aumentada de origem anterior. Este resultado também sugere que o efeito de validade acontece por maior recrutamento cerebral para alvos precedidos por pistas inválidas, atrasando a execução da resposta. Este fenômeno ainda pode ser atribuído à necessidade de monitoramento de conflitos e atualização dos processos de memória operacional. Gómez et al. (2008) não descrevem diferenças entre os RTs a alvos na parte superior e inferior da tela, no entanto, apenas o efeito de validade já esperado para o teste de Posner e também encontrado nos presentes resultados.

Huang, Liang, Xue, Wang, Hu e Chen (2017) pediram que dezesseis participantes saudáveis respondessem a oito alvos circulares dispostos uniformemente ao redor do ponto de fixação precedidos tanto por pistas exógenas, quanto endógenas. Os autores explicaram seus resultados pela redução da incerteza sobre o provável local de aparecimento do alvo, mas demonstraram essencialmente efeito normal de curso temporal da orientação da atenção ao longo de cada tentativa. Isto é, RTs em SOA de 50 e $100 \mathrm{~ms}$ foram significantemente maiores que em outros SOAs como esperado para o teste de Posner, já que os sujeitos tendem a antecipar a resposta ao último SOA, como evidenciado nos resultados aqui apresentados. Com pistas endógenas, Huang et al. (2017) mostraram "redução da incerteza espacial", ou simplesmente, orientação efetiva da atenção, após aproximadamente $200 \mathrm{~ms}$. Pistas exógenas evidenciaram redução da incerteza espacial mesmo em SOAs curtos (menos de $50 \mathrm{~ms}$ ). Os autores também não compararam os RTs entre alvos diametralmente opostos, portanto não especificando qualquer assimetria da orientação encoberta da atenção.

Considerando os resultados dos experimentos anteriores, os resultados do presente experimento adicionam importante contribuição à literatura da área por reafirmar que a orientação da atenção, evidenciada pelo RT aos alvos, não é significativamente assimétrica no plano vertical. Por diferenças metodológicas em relação ao tamanho dos alvos e ao tempo de apresentação dos mesmos durante a tentativa, o presente experimento evidenciou poucos erros por omissão, impedindo comparação com experimentos anteriores da literatura (Rodrigues, 2011) em relação a assimetrias também em relação aos erros por omissão. Ainda assim, expande os resultados anteriores da área (e.g. Castro-Barros et al., 2008; Righi e Ribeiro, 2011).

\section{Considerações Finais}

A orientação encoberta da atenção visual não exibe assimetria vertical, diferentemente da sua manifestação ao espaço horizontal. Esta diferença na manifestação da atenção ao espaço pode estar relacionada à própria forma que exploramos o ambiente, com maior necessidade de monitoramento no campo horizontal em relação ao vertical. Futuramente, importa compreender que regiões neurais promovem orientação encoberta da atenção para os campos visual superior e inferior e, se há assimetria de ativação neural que subsidia a diferença evidenciada nos tempos de reação, como já demonstrado anteriormente na literatura (Corbetta et al., 1993; Nobre et al.,1997 e Kim et al.,1999).

\section{Referências}

Angelopoulou, E., \& Drigas, A. (2021). Working memory, attention and their relationship: A theoretical overview. Research, Society and Development, 10(5), e46410515288. https://doi.org/10.33448/rsd-v10i5.15288

Badzakova-Trajkov, G., Häberling, I. S., Roberts, R. P. \& Corballis, M.C. (2010). Cerebral asymmetries: complementary and independent processes. PLoS One, 5(3):1-9. 
Brain,W.R. (1941). Visual disorientation with special reference to lessions of the right cerebral hemisphere. Brain, 64:244-272.

Castro-Barros, B. A., Righi, L. L., Grechi, G. \& Ribeiro-do-Valle, L. E. (2008). Interlateral asymmetry in the time course of the effect of a peripheral prime stimulus. Brain and Cognition. 66:265-279. doi:10.1016/j.bandc.2007.09.007

Corballis, M. C. (2007). The Evolution of Hemispheric Specializations of the Human Brain. In: Kaas, J.H. (Ed.) Evolution of nervous systems- a comprehensive reference. ed. Academic Press, 4, Cap.22, p.380-394.

Gainotti, G. The role of automatic orienting of attention towards ipsilesional stimuli in non-visual (tactile and auditory) neglect: A critical review. Cortex 46:150-160, 2010. 10.1016/j.cortex.2009.04.006

Gazzaniga, M. (org). (1997). Conversations in the Cognitive Sciences.: MIT Press.

Gómez, C. M., Flores, A. \& Digiacomo, M. R. (2008). Increased brain activation during the processing of spatially invalidly cued targets. The open neuroimaging journal, 2, 106.

Guardiola, A., Ferreira, L. T. C. \& Rotta, N. T. (1998). Associação entre desempenho das funções corticais e alfabetização em uma amostra de escolares de primeira série de Porto Alegre. Arq Neuropsiquiatr, 56, 281-288.

Huang, D., Liang, H., Xue, L., Wang, M., Hu, Q. \& Chen, Y. (2017). The time course of attention modulation elicited by spatial uncertainty. Vision research, $138,50-58$

Hunter, J. D. Matplotlib: A 2D Graphics Environment. Computing in Science \& Engineering. 9(3):90-95. 2007.

James, W. (1890). The principles of Psychology. Vol.1. Henry Holt and Company, NY. Cap. 11, 402-458.

Jewell, G. \& McCourt, M.E. (2000). Pseudoneglect: a review and meta-analysis os performance factors in line bisection tasks. Neuropsychologia. 38:93-110.

Jonides, J. Voluntary versus automatic control over the mind's eye's movement. In: Long, J.B. \& Baddeley, A.D. (Eds.) Attention and Performance IX. Hillsdale, N.J.:Lawrence Erlbaum Associates, 1981. Cap. 11, 187-203.

Kim,Y. H., Gitelman, D. R., Nobre, A. C., Parrish, T. B., LaBar, K. S. e Mesulam, M. M. (1999). The Large-Scale Neural Network for Spatial Attention Displays Multifunctional Overlap But Differential Asymmetry. Neuroimage. 9: 269-277.

Krueger, J. I. \& Heck, P. R. (2019) Putting the P-Value in its Place. The American Statistician, 73(sup1):122-128. 10.1080/00031305.2018.1470033.

Mesulan, M.M. (1999). Spatial attention and neglect: parietal, frontal and cingulated contributions to the mental representation and attentional targeting of salient extrapersonal event. Philosofical Transactions of the Royal Society B. 354:1325-1346.

Nahas, T.R. \& Xavier, G.F. (2004). Atencao. In: Andrade, M., Santos, F.H. e Bueno, O.F.A. (Orgs.) Neuropsicologia Hoje. Cap. 5.

Nobre, A.C., Sebestyen, G. N., Gitelman, D. R., Mesulam, M. M., Frackowiak, R. S. \& Frith, C. D. (1997). Functional localization of the system for visuospatial attention using positron emission tomography. Brain. 120: 515-533.

Peirce, J. W., Gray, J. R., Simpson, S., MacAskill, M. R., Höchenberger, R., Sogo, H., Kastman, E., Lindeløv, J. PsychoPy2: experiments in behavior made easy. Behavior Research Methods. 2019. 10.3758/s13428-018-01193-y.

Posner, M. I. (1980). Orienting os attention. Quartery Journal of Experimental Psychology (B) 32(1):3/25.

Posner, M. I., Cohen, Y., \& Rafal, R. D. (1982). Neural Systems Control of Spatial Orienting. Philosophical Transactions of the Royal Society B: Biological Sciences, 298(1089), 187-198. doi:10.1098/rstb.1982.0081

Rafal, R. D., Posner, M. I., Friedman, J. H., Inhoff, A. W., \& Bernstein, E. (1988). Orienting of visual attention in progres sive supranuclear palsy. Brain, 111(2), 267-280.

Ringo, J.L. et al. (1994). Time is of the essence: A conjecture that hemispheric specialization arises from interhemispheric conduction delay. Cerebral Cortex. 4(4):331-343.

Rodrigues, F. V (2011). Orientação encoberta da atenção visual em não-músicos e músicos com estudo formal em música. 135 f. Tese (Doutorado em Fisiologia Geral) - Universidade de São Paulo, São Paulo - SP.

Sumi, W. Orientação endógena da atenção em ratos por meio de pistas simbólicas. 2011. 69 f. Dissertação (Mestrado)- Universidade de São Paulo, São Paulo- SP.

Wagner, C. J. P. (2003). Atenção visual em crianças e adolescentes: um estudo a partir do paradigma de tempo de reação. 64 f. Dissertação (Mestrado em Psicologia) - Universidade Federal do Rio Grande do Sul. Porto Alegre - RS.

Wasserstein, R. L., \& Lazar, N. A. (2016) The ASA's Statement on p-Values: Context, Process, and Purpose. The American Statistician, 70(2):129-133. 10.1080/00031305.2016.1154108.

Wasserstein, R. L., \& Lazar, N. A. (2019) Moving to a World Beyond “p<0.05”. The American Statistician. 73(sup1):1-19. 10.1080/00031305.2019.1583913. 